\title{
A Novel and One-Pot Synthesis of 6-arylpyrimidin-4-ol
}

\author{
Jitendra M. Gajera, Shital N. Tondlekar, and Laxmikant A. Gharat \\ Drug Discovery Chemistry, Glenmark Research Centre, Glenmark Pharmaceuticals Limited, MIDC, Mahape, \\ Navi Mumbai 400709, India
}

Correspondence should be addressed to Jitendra M. Gajera, jitendrag@glenmarkpharma.com

Received 25 June 2008; Accepted 2 November 2008

Recommended by William Ogilvie

We have developed a novel and one-pot synthesis of 6-arylpyrimidine-4-ol by reacting commercially available alkyl 3-oxo-3arylpropanoate with formamide in the presence of stoichiometric amount of ammonium acetate.

Copyright (C) 2008 Jitendra M. Gajera et al. This is an open access article distributed under the Creative Commons Attribution License, which permits unrestricted use, distribution, and reproduction in any medium, provided the original work is properly cited.

\section{Introduction}

Pyrimidine derivatives are very well known for their various therapeutic applications. Pyrimidine derivatives are used as anticancer [1], anti-HIV [2], antibacterial [3], antimalarial [4], antihypertensive [5], sedative, hypnotics [6], anticonvulsant [7], antithyroid [8], antihistaminic agents [9], and antibiotics [10]. A very recent review describes the significance of pyrimidine derivatives as anti-inflammatory agents [11]. 2-thiopyrimidine derivatives possess potent activity against inflammation and immune disorders [12]. Recently, various pyrimidine derivatives have been reported as vanilloid receptor antagonists [13].

After looking at the diverse properties of pyrimidine derivatives, we selected 4,6-disubstituted pyrimidines as a part of our pharmacophore to synthesize novel antiinflammatory agents. Literature survey revealed that 4,6disubstituted pyrimidines can be prepared using either Biginelli approach [14] or reaction of $\beta$-iminoesters with formamide [15] or reaction of 4,6-dichloropyrimidines with appropriate boronic acids [16]. The only competitive method similar to our approach described in the literature uses formamidine in DMF and affords the product in only $14 \%$ after a reaction time of 3 days [17]. During the course of our research work on synthesis on various 4,6disubstituted pyrimidines, we developed a novel and onepot method for the synthesis of 6-arylpyrimidine-4-ol using commercially available raw materials. The method comprises reaction between 3-oxo-3-arylpropanoate, stoichiometric amount of ammonium acetate, and formamide at elevated temperature.

\section{Results and Discussion}

Various 6-arylpyrimidin-4-ols $\mathbf{3 a - h}$ were prepared by reacting methyl-3-oxo-3-arylpropanoates $\mathbf{1 a - h}$ with formamide in the presence of ammonium acetate with a yield of $50-70 \%$.

The reaction of methyl-3-oxo-3-arylpropanoates 1 with the in situ generated ammonia gives the intermediate methyl 3 -amino-3-arylacrylate 2 , which subsequently reacts with formamide to give 6-arylpyrimidin-4-ols 3 (Scheme 1). One of the intermediates methyl 3-amino-3-phenylacrylate was isolated in $80 \%$ yield and characterized. Isolation and characterization of 2 a confirm the reaction pathway (Table 1 ). The procedure described in the experimental part provides a novel and one-pot approach for the synthesis of 6arylpyrimidin-4-ol.

\section{Experimental}

Commercial solvents and reagents were used without further purification. ${ }^{1} \mathrm{H}$ NMR spectra were recorded on a Varian $300 \mathrm{MHz}$ spectrometer. Melting points are uncorrected. Elemental analysis was performed on a Perkin-Elmer analyzer. Mass spectra were recorded on Thermo Finnigan LCQ DECA XP MAX (ION TRAP) mass spectrometer using atmospheric pressure chemical ionization (APCI) source in positive/negative mode at capillary voltage $3.14 \mathrm{~V}$ and capillary temperature $250^{\circ} \mathrm{C}$. 

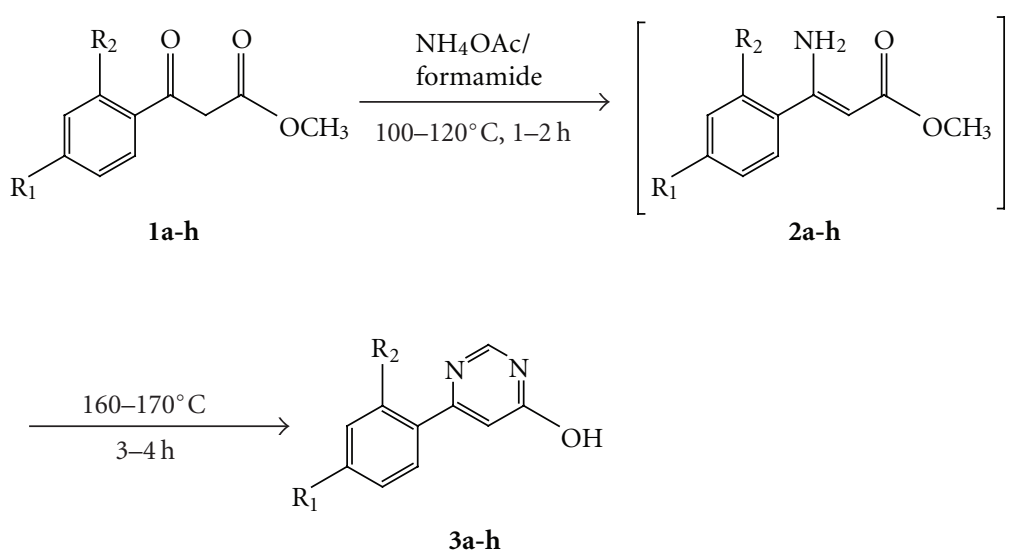

Scheme 1

TABLE 1: Yield and melting point of compounds $3 a-h$ and $2 a$.

\begin{tabular}{lcccc}
\hline Compound & $\mathrm{R}_{1}$ & $\mathrm{R}_{2}$ & Yield (\%) & M. P. $\left({ }^{\circ} \mathrm{C}\right)$ \\
\hline 3a & $\mathrm{H}$ & $\mathrm{H}$ & 65 & $271-272$ \\
3b & $\mathrm{Cl}$ & $\mathrm{H}$ & 70 & $213-215$ \\
3c & $\mathrm{Br}$ & $\mathrm{H}$ & 68 & $311-313$ \\
3d & $\mathrm{F}$ & $\mathrm{H}$ & 61 & $313-315$ \\
3e & $\mathrm{CH}_{3}$ & $\mathrm{H}$ & 57 & $291-292$ \\
3f & $\mathrm{OCH}_{3}$ & $\mathrm{H}$ & 53 & $282-284$ \\
3g & $\mathrm{OCH}_{3}$ & $\mathrm{OCH}_{3}$ & 50 & $252-254$ \\
3h & $\mathrm{H}$ & Cyclopentyloxy & 55 & $260-264$ \\
2a & $\mathrm{H}$ & $\mathrm{H}$ & 80 & - \\
\hline
\end{tabular}

\section{Methyl 3-amino-3-phenylacrylate (2a)}

To a stirred solution of methyl 3-oxo-3-phenylpropanoate $(1 \mathrm{mmol})$ in formamide $(5.0 \mathrm{~mL})$ was added ammonium acetate $(5 \mathrm{mmol})$ at ambient temperature. Reaction mixture was then heated to $110-120^{\circ} \mathrm{C}$ over a period of 1 hour and then held at $110-120^{\circ} \mathrm{C}$ for 1 more hour. It was then cooled to room temperature; diluted with cold water; and extracted with diethyl ether. The residue obtained after removal of diethyl ether was purified through silica gel column using ethyl acetate: petroleum ether $(8: 2)$ as an eluent to give methyl 3-amino-3-arylacrylate $2 \mathrm{a}$ as thick oil. IR ( $\mathrm{KBr})$ : $3445,1622,1491,1320 \mathrm{~cm}^{-1} .{ }^{1} \mathrm{H}$ NMR (DMSO- $\left.d_{6}\right): \delta 3.56$ $(\mathrm{S}, 3 \mathrm{H}) ; 4.78$ (s, 1H); 7.34-7.59 (m, 5H); 7.95 (brs, 2H). $[\mathrm{M}-1]^{-}$: 176.23. Anal. calcd for $\mathrm{C}_{10} \mathrm{H}_{11} \mathrm{NO}_{2}$ : C, 67.78; $\mathrm{H}$, 6.26; N, 7.90. Found: C, 67.65; H, 6.24; N, 7.91.

\section{General Procedure for Preparation of 6-arylpyrimidine-4-ol (3a-h)}

A stirred solution of appropriate methyl 3-oxo-3arylpropanoate 1 ( $1 \mathrm{mmol})$ in formamide $(50 \mathrm{~mol})$ was added ammonium acetate $(5 \mathrm{mmol})$ and heated to 100 $120^{\circ} \mathrm{C}$ over a period of 1 hour and held at $110-120^{\circ} \mathrm{C}$ for 1 more hour. It was then stirred for $4-5$ hours at $160-170^{\circ} \mathrm{C}$. Reaction mixture was cooled to room temperature and diluted with cold water. The precipitated material was extracted with ethyl acetate. The solid product obtained after removal of ethyl acetate was washed with diethyl ether to get pure product.

\section{6-phenylpyrimidine-4-ol (3a)}

This compound was obtained as light yellow solid. IR ( $\mathrm{KBr})$ : $3435,1668,1592,1252,1024 \mathrm{~cm}^{-1} .{ }^{1} \mathrm{H}$ NMR (DMSO- $\left.d_{6}\right): \delta$ $6.87(\mathrm{~s}, 1 \mathrm{H}) ; 7.45-7.47(\mathrm{~m}, 3 \mathrm{H}) ; 8.02-8.03(\mathrm{~m}, 2 \mathrm{H}) ; 8.25$ (s, $1 \mathrm{H}) ; 12.51$ (brs, $1 \mathrm{H})$. MS $[\mathrm{M}+1]^{+}: 172.32$. Anal. calcd for $\mathrm{C}_{10} \mathrm{H}_{8} \mathrm{~N}_{2} \mathrm{O}: \mathrm{C}, 69.76 ; \mathrm{H}, 4.68 ; \mathrm{N}, 16.27$. Found: $\mathrm{C}, 69.74 ; \mathrm{H}$, $4.69 ; \mathrm{N}, 16.31$.

\section{6-(4-chlorophenyl)pyrimidine-4-ol (3b)}

This compound was obtained as light yellow solid. IR $(\mathrm{KBr})$ : $3339,1667,1594,1243,1014 \mathrm{~cm}^{-1} .{ }^{1} \mathrm{H}$ NMR (DMSO- $d_{6}$ ): $\delta 6.91(\mathrm{~s}, 1 \mathrm{H}) ; 7.52(\mathrm{~d}, 2 \mathrm{H}, J=8.4 \mathrm{~Hz}) ; 8.05(\mathrm{~d}, 2 \mathrm{H}, J=$ $8.7 \mathrm{~Hz}) ; 8.26(\mathrm{~s}, 1 \mathrm{H}) ; 12.54(\mathrm{brs}, 1 \mathrm{H})$. MS $[\mathrm{M}+1]^{+}: 207.39$. Anal. calcd for $\mathrm{C}_{10} \mathrm{H}_{7} \mathrm{ClN}_{2} \mathrm{O}: \mathrm{C}, 58.13 ; \mathrm{H}, 3.41 ; \mathrm{N}, 13.58$. Found: C, 58.20; H, 3.42; N, 13.61 .

\section{6-(4-bromophenyl)pyrimidine-4-ol (3c)}

This compound was obtained as light yellow solid. IR $(\mathrm{KBr})$ $3445,1685,1589,1257,1010 \mathrm{~cm}^{-1} .{ }^{1} \mathrm{H}$ NMR (DMSO- $\left.d_{6}\right): \delta$ $6.91(\mathrm{~s}, 1 \mathrm{H}) ; 7.66(\mathrm{~d}, 2 \mathrm{H}, J=9 \mathrm{~Hz}) ; 7.98(\mathrm{~d}, 2 \mathrm{H}, J=8.4 \mathrm{~Hz})$; $8.26(\mathrm{~s}, 1 \mathrm{H}) ; 12.54(\mathrm{bs}, 1 \mathrm{H})$. MS $[\mathrm{M}+1]^{+}: 251.39$. Anal. calcd for $\mathrm{C}_{10} \mathrm{H}_{7} \mathrm{BrN}_{2} \mathrm{O}$ : C, 47.84; $\mathrm{H}, 2.81 ; \mathrm{N}, 11.16$. Found: C, $47.89 ; \mathrm{H}, 2.80 ; \mathrm{N}, 11.15$.

\section{6-(4-fluorophenyl)pyrimidine-4-ol (3d)}

This compound was obtained as light yellow solid. IR ( $\mathrm{KBr})$ 3444, 1672, 1600, 1242, $1035 \mathrm{~cm}^{-1}$. ${ }^{1} \mathrm{H}$ NMR (DMSO- $d_{6}$ ): $\delta 6.88(\mathrm{~s}, 1 \mathrm{H}) ; 7.26-7.31(\mathrm{~m}, 2 \mathrm{H}) ; 8.07-8.11(\mathrm{~m}, 2 \mathrm{H}) ; 8.25$ (s, 1H); 12.52 (brs, $1 \mathrm{H}) .[\mathrm{M}+1]^{+}: 190.97$. Anal. calcd for $\mathrm{C}_{10} \mathrm{H}_{7} \mathrm{FN}_{2} \mathrm{O}: \mathrm{C}, 63.16 ; \mathrm{H}, 3.71 ; \mathrm{N}, 14.73$. Found: $\mathrm{C}, 63.12 ; \mathrm{H}$, 3.70; N 14.76 . 


\section{6-(4-methylphenyl)pyrimidine-4-ol (3e)}

This compound was obtained as light yellow solid. IR ( $\mathrm{KBr})$ : 3422, 1664, 1592, 1254, 1175, $1037 \mathrm{~cm}^{-1} .{ }^{1} \mathrm{H}$ NMR (DMSO$\left.d_{6}\right): \delta 2.34(\mathrm{~s}, 3 \mathrm{H}) ; 6.81(\mathrm{~s}, 1 \mathrm{H}) ; 7.26(\mathrm{~d}, 2 \mathrm{H}, J=7.8 \mathrm{~Hz})$; $7.92(\mathrm{~d}, 2 \mathrm{H}, \mathrm{J}=7.8 \mathrm{~Hz}) ; 8.22$ (s, $1 \mathrm{H}) ; 12.20$ (brs, $1 \mathrm{H}) .[\mathrm{M}+$ $1]^{+}$: 186.39. Anal. calcd for $\mathrm{C}_{11} \mathrm{H}_{10} \mathrm{~N}_{2} \mathrm{O}: \mathrm{C}, 70.95 ; \mathrm{H}, 5.41 ; \mathrm{N}$, 15.04. Found: C, 71.11; H, 5.43; 15.00 .

\section{6-(4-methoxyphenyl)pyrimidine-4-ol (3f)}

This compound was obtained as light yellow solid. IR $(\mathrm{KBr})$ 3397, 1666, 1605, 1245, $1177 \mathrm{~cm}^{-1}$. ${ }^{1} \mathrm{H}$ NMR (DMSO- $d_{6}$ ): $\delta 3.80(\mathrm{~s}, 3 \mathrm{H}) ; 6.77(\mathrm{~s}, 1 \mathrm{H}) ; 7.00(\mathrm{~d}, 2 \mathrm{H}, J=8.7 \mathrm{~Hz}) ; 8.00$ $(\mathrm{d}, 2 \mathrm{H}, J=9 \mathrm{~Hz}) ; 8.21(\mathrm{~s}, 1 \mathrm{H}) ; 12.42$ (brs, $1 \mathrm{H}) .[\mathrm{M}+1]^{+}$: 203.37. Anal. calcd for $\mathrm{C}_{11} \mathrm{H}_{10} \mathrm{~N}_{2} \mathrm{O}_{2}$ : C, 65.34; $\mathrm{H}, 4.98 ; \mathrm{N}$, 13.85. Found: C, 65.38; H, 4.97; N, 13.88 .

\section{6-(2,4-dimethoxyphenyl)pyrimidine-4-ol (3g)}

This compound was obtained as light yellow solid. IR $(\mathrm{KBr})$ $3414,1673,1610,1270,1170 \mathrm{~cm}^{-1}$. ${ }^{1} \mathrm{H}$ NMR (DMSO- $d_{6}$ ): $\delta 3.81(\mathrm{~d}, 6 \mathrm{H}, J=4.5 \mathrm{~Hz}) ; 6.84(\mathrm{~s}, 1 \mathrm{H}) ; 7.02(\mathrm{~d}, 1 \mathrm{H}$, $J=8.4 \mathrm{~Hz}) ; 7.58(\mathrm{~s}, 1 \mathrm{H}) ; 7.65(\mathrm{~d}, 1 \mathrm{H}, J=9 \mathrm{~Hz}) ; 8.20$ (s, $1 \mathrm{H}) ; 12.32$ (brs, $1 \mathrm{H}) .[\mathrm{M}+1]^{+}:$233.36. Anal. calcd for $\mathrm{C}_{12} \mathrm{H}_{12} \mathrm{~N}_{2} \mathrm{O}_{3}$ : C, 62.06; H, 5.21; N, 12.06. Found: C, 62.20; $\mathrm{H}, 5.23 ; \mathrm{N}, 12.04$.

\section{6-[2-(cyclopentyloxy)phenyl]pyrimidine-4-ol (3h)}

This compound was obtained as light yellow solid. IR ( $\mathrm{KBr})$ : $3313,1643,1574,1279,1242 \mathrm{~cm}^{-1}$. ${ }^{1} \mathrm{H}$ NMR (DMSO- $d_{6}$ ): $\delta$ 1.63-1.76 (m, 6H); 1.90-1.99 (m, 2H); $4.95(\mathrm{~m}, 1 \mathrm{H}) ; 6.93$ $(\mathrm{s}, 1 \mathrm{H}) ; 7.00(\mathrm{t}, 1 \mathrm{H}, J=7.8 \mathrm{~Hz}) ; 7.11(\mathrm{~d}, 1 \mathrm{H}, J=8.4 \mathrm{~Hz})$; $7.38(\mathrm{t}, 1 \mathrm{H}, J=6.9 \mathrm{~Hz}) ; 7.93(\mathrm{~d}, 1 \mathrm{H}, J=6.3 \mathrm{~Hz}) ; 8.20$ (s, 1H); 12.42 (brs, $1 \mathrm{H}) .[\mathrm{M}+1]^{+}:$257.13. Anal. calcd for $\mathrm{C}_{15} \mathrm{H}_{16} \mathrm{~N}_{2} \mathrm{O}_{2}$ : C, 70.29; H, 6.29; N, 10.93. Found: C, 70.19; $\mathrm{H}, 6.27$; N, 10.91 .

\section{Acknowledgment}

The authors are thankful to analytical department for analytical support.

\section{References}

[1] E. C. Taylor and B. Liu, "A new and efficient synthesis of pyrrolo[2,3-d]pyrimidine anticancer agents: alimta (LY231514, MTA), homo-alimta, TNP-351, and some aryl 5-substituted pyrrolo[2,3-d]pyrimidines," The Journal of Organic Chemistry, vol. 68, no. 26, pp. 9938-9947, 2003.

[2] P. Herdewijn, J. Balzarini, M. Baba, et al., "Synthesis and anti-HIV activity of different sugar-modified pyrimidine and purine nucleosides," Journal of Medicinal Chemistry, vol. 31, no. 10, pp. 2040-2048, 1988.

[3] J. Matsumoto and S. Minami, "Pyrido[2,3-d]pyrimidine antibacterial agents. 3. 8-alkyl- and 8-vinyl-5,8-dihydro-5oxo-2-(1-piperazinyl)pyrido[2,3-d]pyrimidine-6-carboxylic acids and their derivatives," Journal of Medicinal Chemistry, vol. 18, no. 1, pp. 74-79, 1975.
[4] A. Agarwal, K. Srivastava, S. K. Puri, and P. M. S. Chauhan, "Antimalarial activity and synthesis of new trisubstituted pyrimidines," Bioorganic \& Medicinal Chemistry Letters, vol. 15, no. 12, pp. 3130-3132, 2005.

[5] H. Maeda, T. Akaike, Y. Miyamoto, and M. Yoshida, "Use of 4-amino-6-hydroxypyrazolo[3,4-d]pyrimidine for the manufacture of an antihypertensive agent," European patent no. EP07959298, 1997.

[6] K. S. Jain, T. S. Chitre, P. B. Miniyar, et al., "Biological and medicinal significance of pyrimidines," Current Science, vol. 90, no. 6, pp. 793-803, 2006.

[7] A. K. Gupta, Sanjay, H. P. Kayath, A. Singh, G. Sharma, and K. C. Mishra, "Anticonvulsant activity of pyrimidine thiols," Indian Journal of Pharmacology, vol. 26, no. 3, pp. 227-228, 1994.

[8] J.-F. Lagorce, F. Comby, A. Rousseau, J. Buxeraud, and C. Raby, "Synthesis and antithyroid activity of pyridine, pyrimidine and pyrazine derivatives of thiazole-2-thiol and 2-thiazoline2-thiol," Chemical and Pharmaceutical Bulletin, vol. 41, no. 7, pp. 1258-1260, 1993.

[9] V. Alagarsamy, V. R. Solomon, and M. Murugan, "Synthesis and pharmacological investigation of novel 4-benzyl1-substituted-4H-[1,2,4] triazolo[4,3-a] quinazolin-5-ones as new class of $\mathrm{H}_{1}$-antihistaminic agents," Bioorganic \& Medicinal Chemistry, vol. 15, no. 12, pp. 4009-4015, 2007.

[10] R. L. Tolman, R. K. Robins, and L. B. Townsend, "Pyrrolo[2,3d]pyrimidine nucleoside antibiotics. Total synthesis and structure of toyocamycin, unamycin $\mathrm{B}$, vengicide, antibiotic E212, and sangivamycin (BA-90912)," Journal of the American Chemical Society, vol. 90, no. 2, pp. 524-526, 1968.

[11] M. Amir, S. Javed, and H. Kumar, "Pyrimidine as antiinflammatory agent: a review," Indian Journal of Pharmaceutical Sciences, vol. 69, no. 3, pp. 337-343, 2007.

[12] R. V. Bonnert, P. A. Cage, S. F. Hunt, I. J. S. Walters, and R. P. Austin, "Pteridinone derivatives as modulators of chemokine receptor activity," PCT International Patent Application, WO/2003/024966, 2003.

[13] X. Wang, P. P. Chakrabarti, V. I. Ognyanov, et al., "Trisubstituted pyrimidines as transient receptor potential vanilloid 1 (TRPV1) antagonists with improved solubility," Bioorganic \& Medicinal Chemistry Letters, vol. 17, no. 23, pp. 6539-6545, 2007.

[14] J. Lu, Y. Bai, Z. Wang, B. Yang, and H. Ma, "One-pot synthesis of 3,4-dihydropyrimidin-2(1H)-ones using lanthanum chloride as a catalyst," Tetrahedron Letters, vol. 41, no. 47, pp. 9075-9078, 2000.

[15] F. Zurmühlen, "Process for preparing 5-chloro-4hydroxypyrimidines," US patent no. 5523404, 1996.

[16] E. M. Doherty, J. Zhu, M. Stec, et al., "Amino-pyridine, -pyridine and pyridazine derivatives for use as vanilloid receptor ligands for the treatment of pain," PCT International Patent Application, WO 099284, 2003.

[17] G. T. Wang, S. Wang, R. Gentles, et al., "Amino-substituted heterocycles as isosteres of trans-cinnamides: design and synthesis of heterocyclic biaryl sulfides as potent antagonists of LFA-1/ICAM-1 binding," Bioorganic \& Medicinal Chemistry Letters, vol. 15, no. 1, pp. 195-201, 2005. 


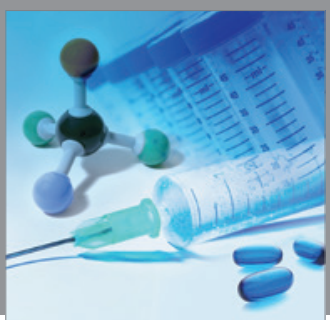

International Journal of

Medicinal Chemistry

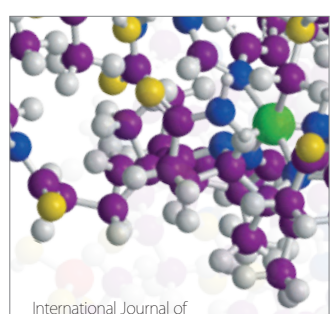

Carbohydrate Chemistry

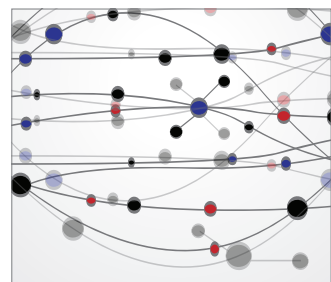

The Scientific World Journal
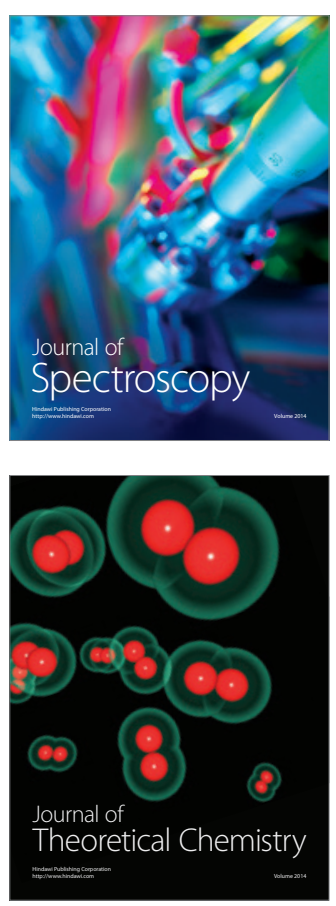
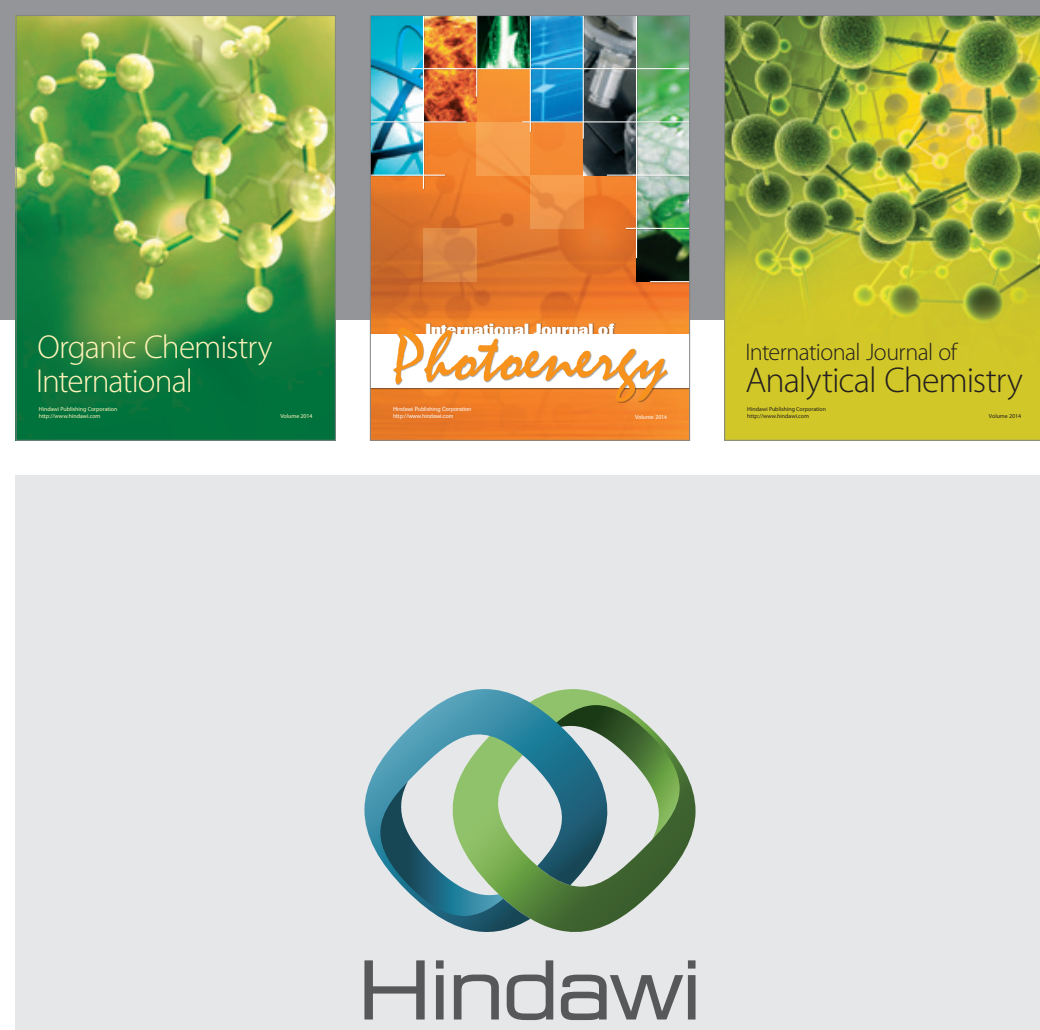

Submit your manuscripts at

http://www.hindawi.com
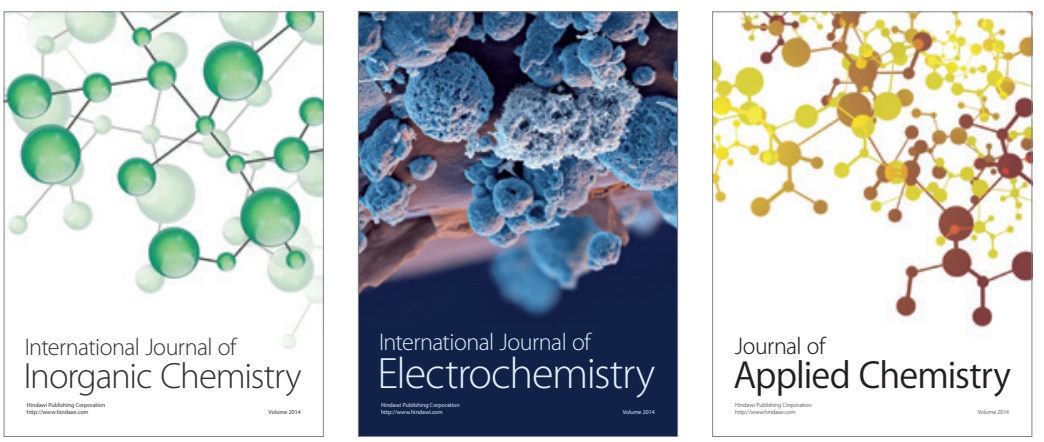

Journal of

Applied Chemistry
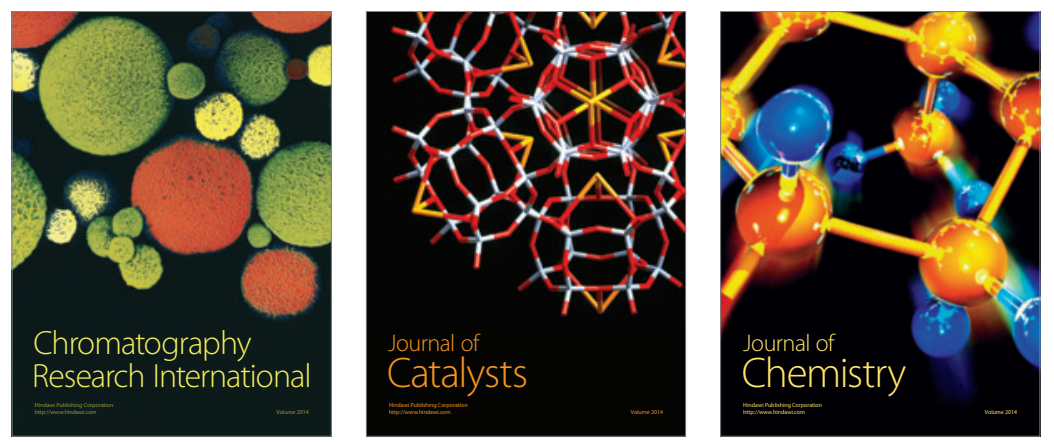
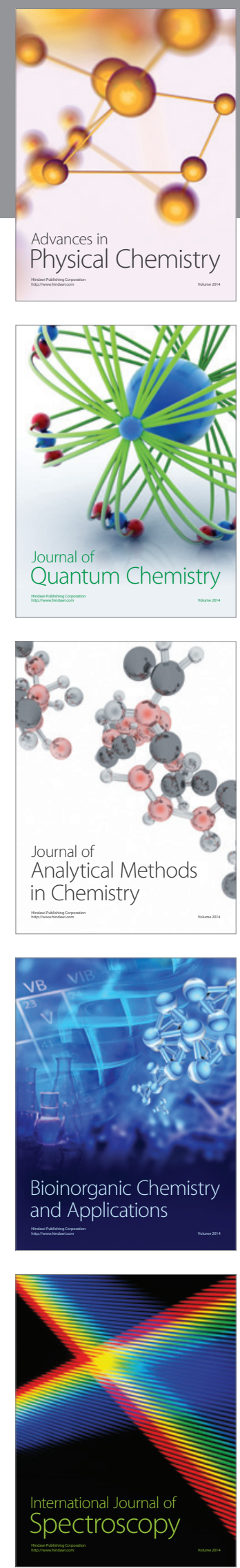